\title{
Computational toolbox for ultrastructural quantitative analysis of filament networks in cryo-ET data
}

\author{
Georgi Dimchev $^{\mathrm{a}, 1}$, Behnam Amiri ${ }^{\mathrm{b}, 1}$, Florian Fäßler ${ }^{\mathrm{a}}$, Martin Falcke ${ }^{\mathrm{b}}$, Florian KM Schur ${ }^{\mathrm{a}, *}$ \\ ${ }^{a}$ Institute of Science and Technology (IST) Austria, Am Campus 1, Klosterneuburg 3400, Austria \\ ${ }^{\mathrm{b}}$ Max Delbrück Center for Molecular Medicine, Robert Rössle Strasse 10, Berlin 13125, Germany
}

\section{A R T I C L E I N F O}

\section{Keywords:}

Actin cytoskeleton

Lamellipodia

Filopodia

Cryo-electron tomography

Image processing

Ultrastructural analysis

\begin{abstract}
A B S T R A C T
A precise quantitative description of the ultrastructural characteristics underlying biological mechanisms is often key to their understanding. This is particularly true for dynamic extra- and intracellular filamentous assemblies, playing a role in cell motility, cell integrity, cytokinesis, tissue formation and maintenance. For example, genetic manipulation or modulation of actin regulatory proteins frequently manifests in changes of the morphology, dynamics, and ultrastructural architecture of actin filament-rich cell peripheral structures, such as lamellipodia or filopodia. However, the observed ultrastructural effects often remain subtle and require sufficiently large datasets for appropriate quantitative analysis. The acquisition of such large datasets has been enabled by recent advances in high-throughput cryo-electron tomography (cryo-ET) methods. This also necessitates the development of complementary approaches to maximize the extraction of relevant biological information. We have developed a computational toolbox for the semi-automatic quantification of segmented and vectorized filamentous networks from pre-processed cryo-electron tomograms, facilitating the analysis and cross-comparison of multiple experimental conditions. GUI-based components simplify the processing of data and allow users to obtain a large number of ultrastructural parameters describing filamentous assemblies. We demonstrate the feasibility of this workflow by analyzing cryo-ET data of untreated and chemically perturbed branched actin filament networks and that of parallel actin filament arrays. In principle, the computational toolbox presented here is applicable for data analysis comprising any type of filaments in regular (i.e. parallel) or random arrangement. We show that it can ease the identification of key differences between experimental groups and facilitate the in-depth analysis of ultrastructural data in a time-efficient manner.
\end{abstract}

\section{Introduction}

Cryo-electron tomography (cryo-ET) provides one route to obtain high-resolution insights into natively preserved biological environments in cells and tissues. Beyond its use for in situ structure determination (reviewed by (Schur, 2019; Zhang, 2019)), its main strength lies in its ability to provide contextual information for the molecules under study, such as higher-order arrangements of proteins within cells. This information can be linked to functional data to provide a holistic quantitative description of cellular processes. In this regard, cryo-ET, with its resolution on the level of individual molecules, is well-positioned to complement experimental data obtained by other modalities, such as genetic perturbation experiments or light-microscopy imaging.

One major challenge in cryo-ET is the extraction of statistically relevant quantitative parameters from sufficiently large datasets. Several inherent attributes of the method impede large-scale analysis, including the low signal-to-noise ratio (SNR) in tomograms, the complexity of cellular data, and the need for appropriate computational tools to extract meaningful biological data. Hence, while the potential of cryo-ET as a qualitative method is commonly accepted for applications where the analysis of a few tomograms is sufficient to detect and describe novel subcellular features, its potential as a quantitative technique to compare subtle differences among genetically distinct samples is not yet fully realized.

Recent improvements in cryo-EM sample preparation (Buckley et al., 2020; Engel et al., 2019; Fäßler et al., 2020c; Toro-nahuelpan et al., 2019; Zachs et al., 2020), automated EM data acquisition (Bouvette et al., 2021; Chreifi et al., 2019; Eisenstein et al., 2019; Schorb et al.,

\footnotetext{
* Corresponding author.

E-mail address: florian.schur@ist.ac.at (F.K. Schur).

1 Equal contribution.
} 
2019), image processing workflows (Chen et al., 2019; Mastronarde and Held, 2017), and data analysis allow the evaluation of large datasets and comparison of various in situ features between multiple experimental conditions. These improvements, although very suitable for being combined with the nowadays relatively straightforward genetic manipulation of cell lines via CRISPR/Cas9 techniques, are yet to be routinely applied in workflows that facilitate the high-throughput analysis and comparison of ultrastructural characteristics between genetically modified cell lines. Studying such large datasets is a prerequisite to compensate for random errors that can occur when segmenting and vectorizing objects in tomograms. Thus, the accuracy of the obtained data ultimately depends on the quality of the tomograms and the dataset size, where the latter can compensate for tomogram-specific errors (i.e., caused by local variations in tomogram quality).

The characterization of molecular machineries underlying cell migration strongly benefits from quantitative descriptions. This is particularly true for the actin cytoskeleton and its associated regulatory proteins (Blanchoin et al., 2014). Together, they form dynamic higherorder structures at the leading edge of migrating cells including sheetor finger-like protrusions, such as lamellipodia, or microspikes and filopodia, respectively. The ultrastructural and morphological characterization of these assemblies in wild type or genetically modified cells, combined with experiments elucidating cellular dynamics, can provide an accurate description of the role of selected players in the initiation and maintenance of actin networks or how actin filaments produce forces in a variety of cellular mechanisms (Akamatsu et al., 2020; Fäßler et al., 2020b; Kage et al., 2017).

(Cryo-) electron tomography has provided ultrastructural insights into distinct actin filament assemblies, such as lamellipodia, filopodia, actin waves or pathogen-mediated filament networks (Damiano-Guercio et al., 2020; Jasnin et al., 2013; Kage et al., 2017; Mueller et al., 2017; Urban et al., 2010; Weber et al., 2019; Winkler et al., 2012). Specifically, major progress was achieved by introducing computational tools to vectorize filaments, either based on template matching, reduced representation templates or using the localized radon transform, to then derive parameters for entire filament networks (Anderson et al., 2017; Rigort et al., 2012; Volkmann, 2004; Winkler et al., 2012; Xu et al., 2015). Due to the experimental complexity, previous studies analyzed datasets ranging from a few to $\sim 30$ tomograms (Jasnin et al., 2019, 2016, 2013; Mueller et al., 2017; Vinzenz et al., 2012), and the subsequent quantitation of the vectorized filament information employed single-function customized scripts predominantly to derive a limited number of parameters. However, given the ongoing developments in the cryo-ET field, theoretically, datasets with hundreds of tomograms can be acquired within a few days. An exhaustive quantitative analysis could reveal more detailed descriptions of the mechanisms underlying actin network assembly and maintenance, but requires facilitated analysis workflows that are also more easily applicable to the growing base of researchers using cryo-ET approaches.

We have developed a MATLAB-based analysis toolbox, which is to be used after any filament vectorization approach of choice, that enables the semi-automatic quantification of filamentous networks from large cryo-ET datasets. It allows for pre-processing coordinate information of filaments derived from segmented tomograms, advanced visualization of whole structures and extraction of a large number of ultrastructural parameters as either numerical values or as figures and plots. Furthermore, the toolbox facilitates cross-comparison of experimental conditions. We demonstrate the feasibility of this workflow by comparing differentially manipulated lamellipodial actin networks, parallel actin filament arrays in protruding filopodia or non-protruding microspikes in extracted and fixed adherent cells, and validated our toolbox in a tomogram of a previously published dataset containing thin and thick filaments in sarcomeres of neonatal rat cardiomyocytes (Burbaum et al., 2021).

\section{Results and discussion}

\subsection{A computational toolbox facilitating ultrastructural analysis of filament-rich structures}

To facilitate the adoption of a more streamlined ultrastructural analysis approach of filament populations and their characteristics in cryo-electron tomograms, we designed our computational toolbox with four key aspects in mind:

1) Compatibility: Our toolbox is implemented to analyze vectorized filaments, thus allowing the user to employ their own method of choice to generate coordinate files of filaments from cryo-ET data (Fig. 1A). Examples for such workflows are given below: Tomograms can be preprocessed prior to vectorization using tools based on Deep learning, such as YAPiC (https://yapic.github.io/yapic/), to segment filaments and increase the SNR. Filament vectorization can then be performed using available tools based on a template matching approach (Rigort et al., 2012), as implemented in the commercial software Amira-Avizo (Thermo Fisher Scientific), in MATLAB-scripts using the localized Radon transform (Winkler et al., 2012) (Suppl. Fig. 1) or via manual filament tracking (for example in IMOD). Importantly, our toolbox is blind towards prior data vectorization approaches and requires as input the extracted filament coordinate data solely in tab-delimited format, where four columns describe the filament/object identifier and the $\mathrm{x}, \mathrm{y}$ and $\mathrm{z}$ coordinates, respectively. Such format can be easily obtained from the published vectorization software workflows or IMOD after manual filament tracking.

2) User-friendliness \& versatility: We developed our computational toolbox to require minimal MATLAB proficiency and no prior coding experience. Several graphical user interfaces (GUIs) guide the user through extracting outputs from large datasets in a time-efficient manner (Fig. 1B). Specifically, we have compiled the extraction of multiple predefined ultrastructural parameters from different filament architectures, such as either randomly distributed networks (e.g., lamellipodia) or quasi-parallel or bundled filaments (e.g., filopodia/ microspikes) into one GUI-based step. A summary of all parameters is provided in Table 1 and 2 (see also Methods section for their mathematical descriptions). These customized parameters describe whole structural features, filament ultrastructural characteristics, as well as physical properties. An exhaustive documentation file and test data are provided with the toolbox, and guide the user through the individual steps and provide in-depth details on their use.

3) Data curation: Cryo-ET data inherently suffers from low SNR and other experimental limitations that warrant caution when processing and analyzing tomograms. Importantly, data quality optimization should be best performed upstream of our toolbox, e.g. via optimized data acquisition strategies, individualized approaches for tomogram filtering or when selecting the appropriate software and associated settings for filament vectorization. For example, the influence of the missing wedge can complicate the detection of filaments orthogonal to the tilt axis (for the influence of the missing wedge on actin filaments of various orientations see Supplementary Figure 1 in (Jasnin and Crevenna, 2016)), which can be potentially overcome using dual-axis tomography (Mastronarde, 1997; Winter and Chlanda, 2021). In section 2 of the supplemental user manual, we have provided several suggestions on key steps to optimize and on how to avoid issues with tracking and segmentation. However, the low SNR and missing wedge in cryo-ET data often cause unwanted artifacts which are difficult to remove during any upstream acquisition and processing step, and which, upon deriving coordinate files, result in false-positive filament tracking (Fig. 1C, Suppl. Fig. 2A).

In order to reduce such false-positive information in downstream analysis, we implemented data cleaning and curation options to remove vector data of unspecific structures and background. Specifically, we implemented filtering of data files by custom ranges for filament length, angular distribution, or bendiness (Suppl. Fig. 2A). The results of the 

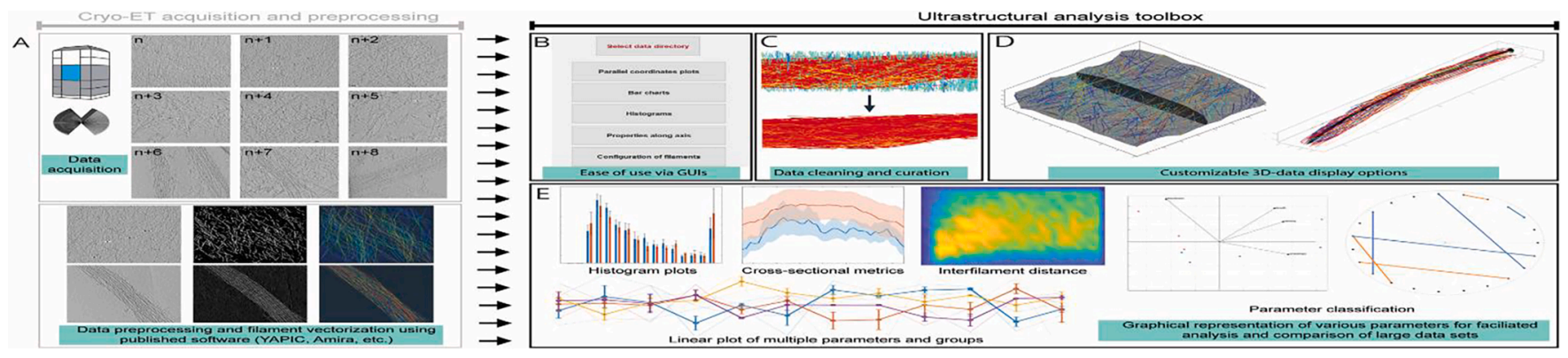

Fig. 1. Workflow for the ultrastructural characterization of filamentous assemblies. (A) Tilt-series acquisition and tomogram reconstruction of datasets of variable size (upper panel) is followed by filament vectorization and extraction of filament coordinates data (lower panel). The extracted filament coordinate files can then be used as analysis input in the computational toolbox. Please note that steps described in (A) are not part of the presented toolbox. (B-E) Examples of tools and options available in the computational analysis toolbox. (B) GUI-based modules of the toolbox facilitate the manipulation and analysis of datasets. (C) Data cleaning based on user-defined ranges for filament length, bendiness or angular distribution in X/Z-axis, allows for the removal of unspecific background or exclusion of filament populations with common characteristics. (D) A 3D visualization module allows user-defined specific representation of sample characteristics. (E) Several examples of the available graph and plot types for the facilitated data analysis of a large number of ultrastructural characteristics. Further details and specific examples for these plots are provided in Fig. 2(D-F), Fig. 4(B-D), Fig. 4(F-H) and Fig. 5C.

cleaning steps can be fed into the visualization module integrated in the toolbox to receive feedback upon testing various parameters. Since the input and output format of the cleaned coordinates is also compatible with IMOD, an iterative manual manipulation of model files or cleaning of individual artifacts in IMOD and data analysis within the MATLABbased toolbox is possible. To allow for the comparison of datasets acquired with different pixel sizes or fields of view (FOV), we have included an option to define pixel size and re-scale the dimensions of coordinate files in a semi-automated fashion. This enables the normalization of non-uniform datasets to compare differently acquired experimental groups (Suppl. Fig. 2B).

4) Simplified data interpretation and classification: We have integrated a GUI-based data visualization module, which works seamlessly with the output of the analysis scripts (Fig. 1D, Suppl. Fig. 3A). It allows reviewing the quality of processed data using various instruments, such as color-coding of filaments by customized parameter ranges (Suppl. Fig. 3B), displaying cross-sections along the axis, as well as overlaying $3 \mathrm{D}$ objects to extract representative images containing sufficient visual information (Suppl. Fig. 3C). We facilitate the display of data and group comparisons by allowing to select the desired outcome through the user interface. Experimental groups can easily be assigned, compared visually by multiple types of readily available graphs, correlated to each other or classified via PCA analysis (Fig. 1E). The output of the analysis is also saved in .xls file format to allow straightforward extraction of raw parameter values for various statistical tests or to feed them in other software workflows.

\subsection{Data analysis with the computational toolbox}

In order to demonstrate the potential of the computational toolbox and its ability to dissect ultrastructural data and quantify differences between experimental groups, we compared distinctly organized branched networks or bundled arrays of filaments in vitreously frozen B16-F1 melanoma cells (Fig. 2A and B; Fig. 3). To this end, we acquired cryo-electron tomograms of B16-F1 melanoma cells under different conditions. Cells were fixed and extracted as described previously (Fäßler et al., 2020a) in order to preserve lamellipodia, filopodia and microspikes, while at the same time enhancing contrast due to the removal of membrane and cytosolic proteins. Following vitrification and data acquisition, filament coordinates were obtained with either the filament segmentation package in the Amira software or a combination of deep-learning with the YAPiC software-based segmentation of filaments, followed by filament tracking via MATLAB scripts using the localized Radon transform (Winkler et al., 2012). Both approaches can result in similar outcomes (Suppl. Fig. 1). However, since obtaining filament coordinate information in Amira required less manual user- defined parameter testing, increased throughput and also resulted in higher filament density, we decided to perform the remaining analysis presented in this manuscript using filament coordinates derived from Amira. We note that segmentation using a convolutional neural network (CNN) like YAPiC can be applied in combination with any filament tracking approach.

\subsection{Analysis of branched actin networks}

The Arp2/3 complex is an integral component in dendritic actin networks. It binds to preexisting actin (mother) filaments, promotes the nucleation of new (daughter) filaments and thereby forms characteristic branch junctions, which link mother and daughter filaments (Molinie and Gautreau, 2018). We analyzed either untreated (DMSO-control) B16-F1 cells (Fig. 2A, Suppl. Fig. 4A) or B16-F1 cells treated with the Arp2/3 complex inhibitor CK666 ( $\sim 10 \mathrm{~min} ; 210 \mathrm{uM}$ concentration) (Fig. 2B, Suppl. Fig. 4B) to compare branched filament networks with different architecture. CK666 binds to the Arp2/3 complex and inhibits actin filament nucleation by stabilizing the inactive state of the complex, thus also inhibiting dendritic actin network formation (Hetrick et al., 2013). As reported previously, CK666 treatment led to the rearrangement of filaments, manifested by changes in their angular distribution and density in comparison to untreated cells (Henson et al., 2015). While this is already discernible in the tomographic data, it becomes even more evident when the filament tracks are plotted and colored by ranges of angular distribution relative to the cell edge using our computational toolbox color-coding option (Suppl. Fig. 4B). For an initial comparison of datasets, our toolbox uses a linear visualization plot for all parameters describing filamentous networks/lamellipodia (Fig. 2C, Tables 1 and 2). This type of graph provides a convenient and fast approach to identifying the key differences between experimental groups, which can then be analyzed in detail with more specialized visualization options. For instance, filament density between control and CK666-treated cells can be averaged and traced along the axis of the entire structure (Fig. 2D) to identify potential differences between front and back regions of the structure. Other parameters, such as average lamellipodium height, are easily discerned by plotting them in a bar chart (Fig. 2E). Histogram plots can be used to compare the distribution of parameter values between experimental groups. In the presented case, this analysis confirms the visual impression of an increased fraction of filaments in CK666-treated cells, running in angles of $>60$ degrees to the cell edge, relative to control cells (Fig. 2F). All parameters shown in Fig. 2C can be displayed with various plots in order to separate filament populations in bins of custom size, to discover potential differences in their values along the axis between two or more experimental conditions, to find correlations or to categorize data of sufficiently large size 
Table 1

Description of parameters included in the computational toolbox, specific to either quasi-parallel filament arrays or dendritic networks.

\begin{tabular}{|c|c|c|}
\hline \multicolumn{3}{|c|}{$\begin{array}{l}\text { Parameter set describing entire quasi-parallel filament structures(e.g. filopodia/ } \\
\text { microspikes) }\end{array}$} \\
\hline Parameter name & $\begin{array}{l}\text { Parameter short description and biological } \\
\text { relevance }\end{array}$ & $\begin{array}{l}\text { Unit [value } \\
\text { ranges] }\end{array}$ \\
\hline Length (of structure) & $\begin{array}{l}\text { The axis length of the filopodium/ } \\
\text { microspike. }\end{array}$ & $\mathrm{nm}$ \\
\hline $\begin{array}{l}\text { Bendiness (of } \\
\text { structure) }\end{array}$ & $\begin{array}{l}\text { Bendiness is defined as the ratio of the total } \\
\text { axis length and its end-to-end distance. } \\
\text { Bendiness of } 1 \text { defines a straight line. The } \\
\text { more bent the structure, the higher the } \\
\text { value. }\end{array}$ & $\begin{array}{l}\text { a.u./ratio } \\
\quad[\geq 1]\end{array}$ \\
\hline $\begin{array}{c}\text { Bending energy } \\
\text { density (of } \\
\text { structure) }\end{array}$ & $\begin{array}{l}\text { A metric of curvature and bending property } \\
\text { of a contour, such as the axis of a } \\
\text { filopodium/microspike. Bent structures } \\
\text { have a higher value of bending energy. As } \\
\text { compared to the bendiness parameter } \\
\text { (described above), higher values of bending } \\
\text { energy can also reflect various contour } \\
\text { anomalies, such as edges or sharp change in } \\
\text { directionality/curvature of a filament. For a } \\
\text { more detailed mathematical description, see } \\
\text { methods section. }\end{array}$ & $\mathrm{nm}^{-2}$ \\
\hline $\begin{array}{l}\text { Cross-sectional } \\
\text { circularity (of } \\
\text { structure) }\end{array}$ & $\begin{array}{l}\text { Mean cross-sectional circularity of the } \\
\text { structure averaged across a user-defined } \\
\text { number of equidistant cross-sections along } \\
\text { the axis. Circularity is defined as the ratio } \\
\text { of the cross-sectional area to the area of a } \\
\text { circle with the same perimeter. It } \\
\text { characterizes how similar the average cross } \\
\text { section of the structure is to a perfect circle. } \\
\text { For a perfect circular cross-section } \\
\text { circularity equals } 1 \text {, while more flattened } \\
\text { structures will have a lower value of this } \\
\text { parameter. For a more detailed mathematical } \\
\text { description, see methods section. }\end{array}$ & $\begin{array}{l}\text { a.u./ratio } \\
\quad[\leq 1]\end{array}$ \\
\hline $\begin{array}{c}\text { Vertical bending } \\
\text { stiffness (of } \\
\text { structure) }\end{array}$ & $\begin{array}{l}\text { The moment of inertia of filaments in a } \\
\text { cross-section with respect to the y-axis. The } \\
\text { parameter is associated with stiffness and } \\
\text { describes the resistance of the structure } \\
\text { against bending in z direction (e.g. } \\
\text { filopodium tip rises from the substrate). } \\
\text { Note that the parameter does not consider } \\
\text { potential binding events between } \\
\text { individual filaments or between filaments } \\
\text { and other proteins, which might occur in } \\
\text { situ. For a more detailed mathematical } \\
\text { description, see methods section. }\end{array}$ & $\mathrm{nm}^{2}$ \\
\hline $\begin{array}{l}\text { Lateral bending } \\
\text { stiffness (of } \\
\text { structure) }\end{array}$ & $\begin{array}{l}\text { The moment of inertia of the filaments in a } \\
\text { cross-section with respect to z-axis. The } \\
\text { parameter is associated with stiffness and } \\
\text { describes the resistance against lateral } \\
\text { bending, e.g. how resistant a filopodium/ } \\
\text { microspike is to bending sideways along its } \\
\text { axis. Note that the parameter does not } \\
\text { consider potential binding events between } \\
\text { individual filaments or between filaments } \\
\text { and other proteins, which might occur in } \\
\text { situ. For a more detailed mathematical } \\
\text { description, see methods section. }\end{array}$ & $\mathrm{nm}^{2}$ \\
\hline \multicolumn{3}{|c|}{$\begin{array}{l}\text { Parameter set describing entire network structures of randomly distributed filaments } \\
\text { (e.g. lamellipodia) }\end{array}$} \\
\hline Parameter name & $\begin{array}{l}\text { Parameter short description and biological } \\
\text { relevance }\end{array}$ & Unit \\
\hline Height (of structure) & $\begin{array}{l}\text { Average height of the structure. It is } \\
\text { averaged for a user-defined number of } \\
\text { equidistant cross-sections, considering the } \\
\text { lowest and highest Z-coordinate points in } \\
\text { each cross-section. }\end{array}$ & $\mathrm{nm}$ \\
\hline
\end{tabular}

Table 2

Description of parameters included in the computational toolbox, valid for both quasi-parallel filament arrays and dendritic networks.

Parameter set describing filaments in both quasi-parallel and randomly oriented networks (filopodia/microspikes/lamellipodia)

\begin{tabular}{|c|c|c|}
\hline Parameter name & $\begin{array}{l}\text { Parameter short description and biological } \\
\text { relevance }\end{array}$ & Unit \\
\hline Length of filaments & $\begin{array}{l}\text { Mean contour length of all filaments in the } \\
\text { entire structure. }\end{array}$ & $\mathrm{nm}$ \\
\hline $\begin{array}{l}\text { Bendiness of } \\
\text { filaments }\end{array}$ & $\begin{array}{l}\text { Mean bendiness of the filaments averaged } \\
\text { within the entire structure. The bendiness } \\
\text { parameter for filaments is derived similarly } \\
\text { to the one for the entire structure (see } \\
\text { Table 1). }\end{array}$ & $\begin{array}{l}\text { a.u./ratio } \\
\quad[\geq 1]\end{array}$ \\
\hline
\end{tabular}

Barbed/pointed ends Density of filament start/end points along

$\mathrm{um}^{-3}$ the axis of the structure, also known as pointed/barbed ends, respectively, for actin filaments. The pointed end of a filament is defined as the one closer to the base of the structure, while the barbed end as the one closest to the edge/tip.

Anisotropy of $\quad$ Measures the preference of certain filament orientations towards the edge/tip. For a filament network of entirely random orientations, anisotropy is zero. For a structure with preferred angle of e.g. 70 degrees to the edge, anisotropy is higher. For bundled filament structures with parallel filament arrays, such as in filopodia, the value of the parameter is maximal. For $a$ more detailed mathematical description, see methods section.

Angle of filaments Mean angle of the end-to-end vector of filaments to the reference direction, averaged across the whole actin structure.

Volume fraction of Ratio of the total volume of all filaments to filaments the total volume of the structure. For a more detailed mathematical description, see methods section.

Bending energy A metric of filament curvatures averaged density of filaments within the entire structure. The bending energy density for filaments is derived similarly to bending energy density of structure (see Table 1). For a more detailed mathematical description, see methods section.

Number of filaments Total number of individual filaments in the in the structure structure.

Cross-sectional area Mean cross-sectional area of the structure averaged for a user-defined number of equidistant cross-sections along the axis.

$$
\begin{gathered}
\text { Cross-sectional } \\
\text { density }
\end{gathered}
$$

Ratio of the number of filaments passing a cross-section to the area of the cross-section, averaged for a user-defined number of equidistant cross-sections along the axis.

Cross-sectional volume fraction Ratio of the total cross-sectional area of filaments passing a cross-section to the area of the cross-section, averaged for multiple cross-sections along the axis. For a more detailed mathematical description, see methods section.
Cross-sectional number of filaments Number of filaments passing a cross-section, averaged for a user-defined number of equidistant cross-sections along the axis.

Additional parameters Base/tip ratio of

The value of Parameter $X$ in the first half of Parameter X the structure (closer to the base) divided by the mean value of the Parameter in the second half of the structure (closer to the tip/edge).

by e.g., PCA analysis (see Fig. 1E for example).

In addition, our toolbox allows the analysis of the distribution of filament start and end points along the structure axis. This type of analysis could reveal potential differences in the density of actin filaments pointed/barbed ends in back vs. middle vs. front regions of 
A
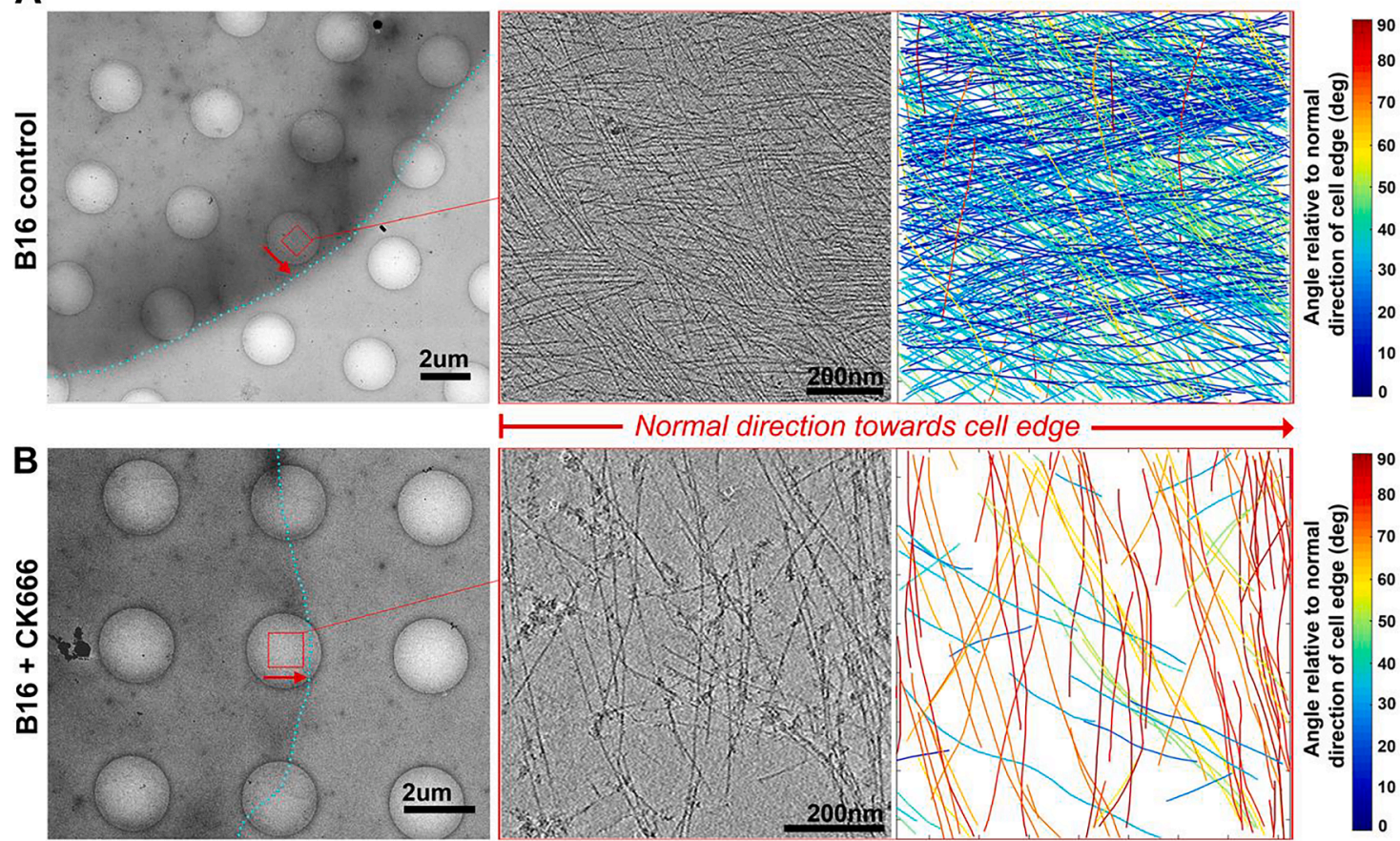<smiles>CC</smiles>
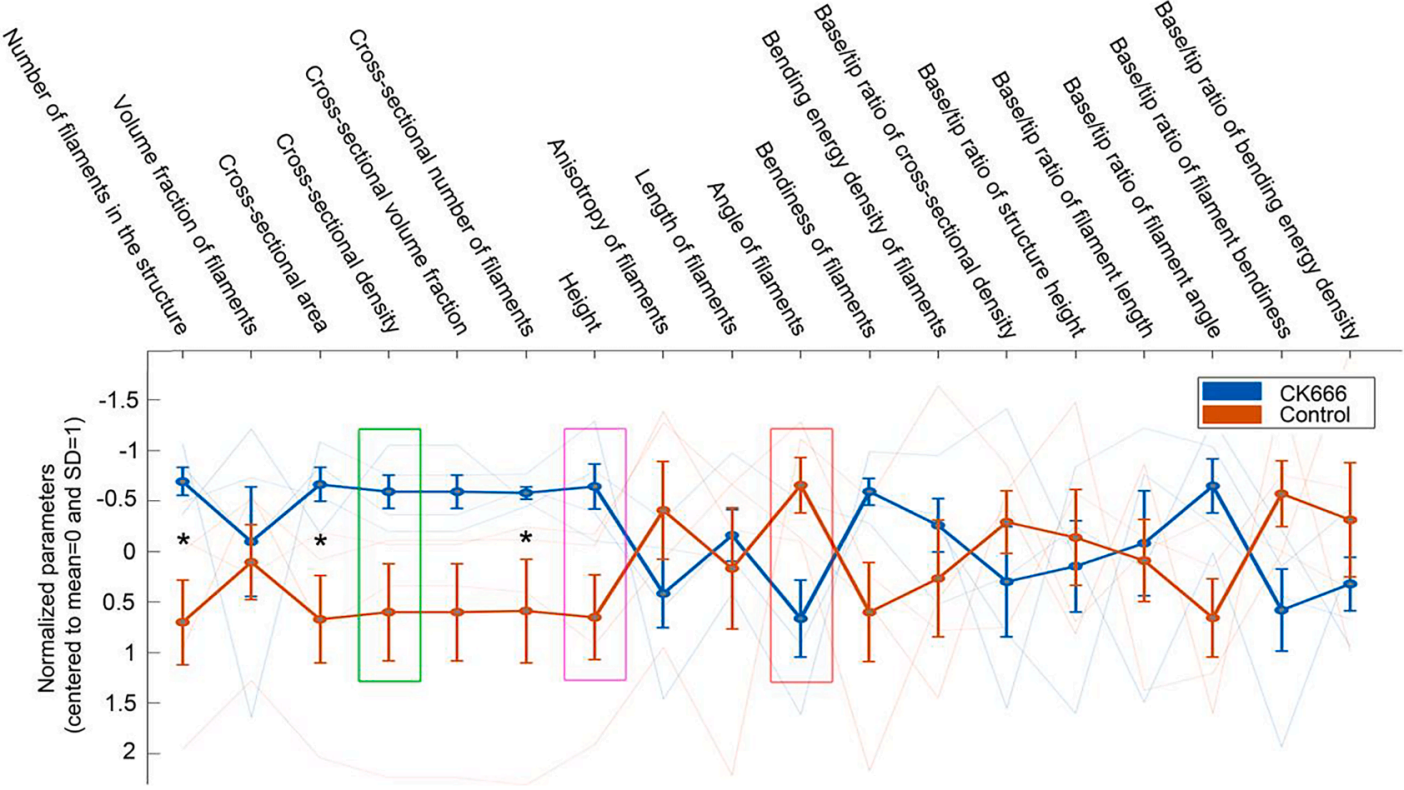

D

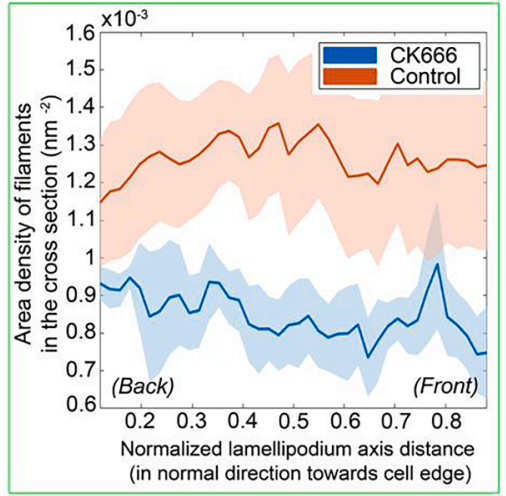

E

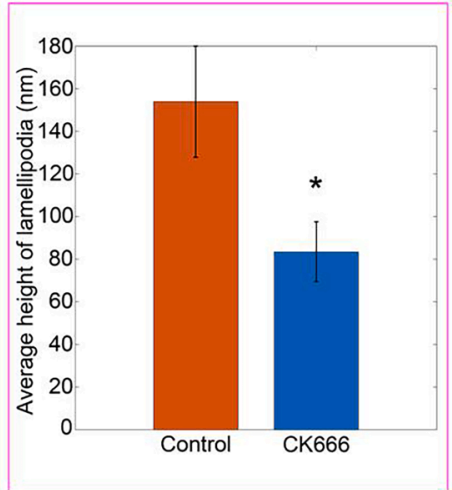

F

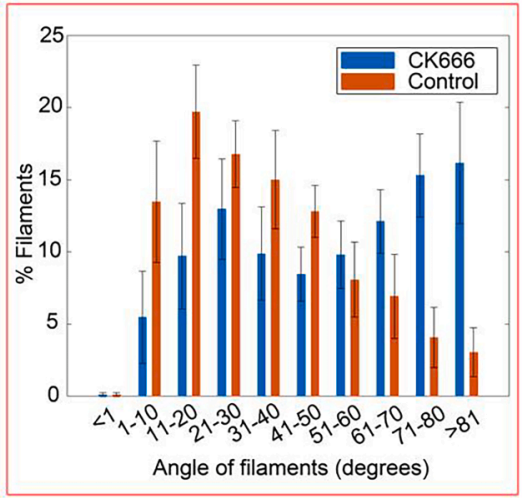


Fig. 2. Analysis of lamellipodial networks. (A-B) Analysis of filament networks in cryo-electron tomograms of lamellipodia of extracted and fixed (A) B16-F1 mouse melanoma cells treated with DMSO control or (B) treated with 210uM of the Arp2/3 inhibitor CK666. Left panels: Low-magnification overview images showing the cell periphery of the respective cells. The cell edges are manually annotated by a cyan dotted line to aid the reader. Middle panels: 10 summed computational slices through bin8-tomograms of B16 lamellipodia. Their positions at the cell periphery are annotated by red boxes in the left panel. Right panels: Visual output generated by the computational toolbox, color coded for angular distribution relative to the normal direction of the cell edge. Red arrows indicate the orientation of the axis towards the lamellipodial edge, i.e. normal direction. Scale bar sizes are annotated in the figure. (C) Normalized quantitative values of multiple parameters, plotted linearly in one graph, can reveal differences in ultrastructural characteristics between experimental treatments. Thick lines indicate the averaged values for all data files in a group, while faint lines show the averaged (when appropriate) values of individual data files for every parameter. Information on the individual parameters is given in Tables 1 and 2. Please note the orientation of the Y-axis. (D-F) Parameters selected from the linear graph in panel (C) plotted individually. (D) Cross-sectional filament density along the lamellipodial axis in normal direction to the cell edge. The transparent outlines indicate standard deviation. (E) Average lamellipodium height. (F) Angular orientation of filaments in lamellipodia (bin number and size is customizable). All plot options are easily accessible via a GUI-based module. Statistical significance (paired $t$-test, $\mathrm{p} \leq 0.05$ ) between experimental groups is marked with *. $\mathrm{N}$ of tomograms is 4 for both control and CK666 groups. (For interpretation of the references to color in this figure legend, the reader is referred to the web version of this article.)
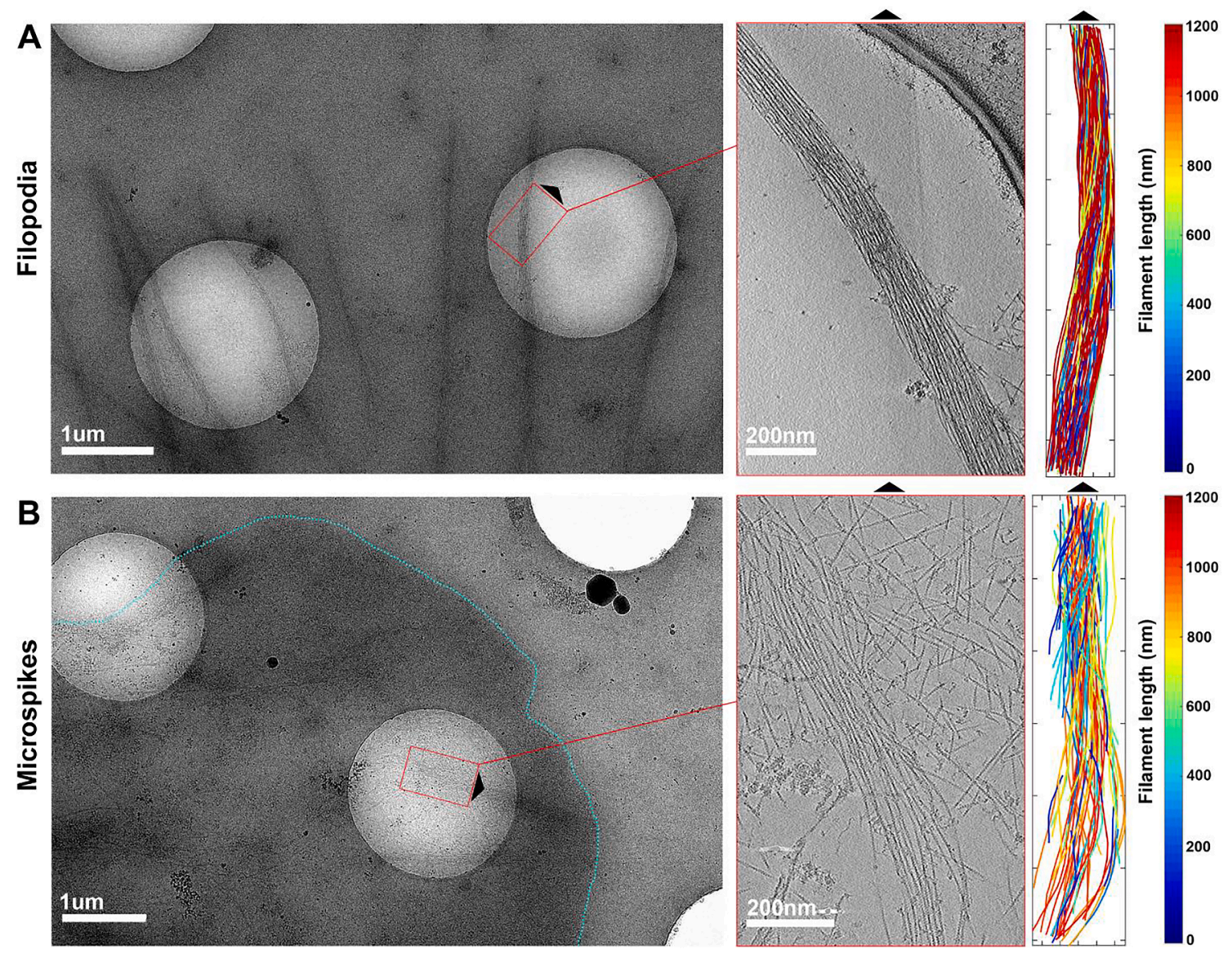

Fig. 3. Representative EM micrographs of filopodia and microspikes used for analysis. In order to demonstrate the feasibility of the computational toolbox workflow in distinguishing quantitative ultrastructural differences between experimental groups containing quasi-parallel filaments, we compared (A) filopodia protruding beyond the cell edge to (B) posterior regions of microspikes embedded within lamellipodia. Filaments belonging to the lamellipodial networks were manually removed with IMOD. In (A) and (B), left panels show medium magnification maps of the cell periphery. The cell edge is manually annotated by a cyan dotted line, to aid the reader. Analyzed regions are highlighted with red rectangles; middle panels show a representative tomogram slice of the analyzed region; right panels show the visual output generated by the computational toolbox, color coded for filament length. Black arrowheads indicate the direction of the microspike/ filopodial tip. (For interpretation of the references to color in this figure legend, the reader is referred to the web version of this article.)

lamellipodia for each experimental condition, assuming that the pointed end of a filament is the one closer to the base and the barbed end the one close to the edge/tip of the structure (Suppl. Fig. 5). An important consideration is the potential accumulation of these ends on the edges of the segmentation and hence the avoidance of including false-positives into the final analysis (Suppl. Fig. 5A). We address this by allowing the user to set boundaries for selecting pointed/barbed ends lying in defined sections along the axis of the structure (i.e., away from the edge of the selected area) (Suppl. Fig. 5B) or within certain distance ranges from the base or away from the tip of the structure. Another important consideration is that this type of analysis is based on the assumption of filament polarity, which in many structures cannot be easily verified. Thus, this parameter remains useful for approximating the distribution of filament ends in proximity to either the base or the tip of the structure, but unless combined with other approaches of actin filament polarity detection (for published examples see (Martins et al., 2021; Narita et al., 2012)), it cannot be used for drawing definitive conclusions on filament polarity.

\subsection{Analysis of bundled actin filament arrays}

We used our toolbox for examining ultrastructural characteristics of 
bundled filament structures and compared protruding filopodia with posterior regions of non-protruding microspikes (Fig. 3). Posterior regions show a less uniform arrangement of filaments and are often diverging or splayed apart (as previously reported in (Svitkina et al., 2003)). On the contrary, protruding filopodia are characterized by more tightly bundled filaments (Suppl. Fig. 6). Similar to the above-described approach for analysis of branched networks, the toolbox enables displaying all parameters associated with bundled filament arrays in a linear plot (Fig. 4A), where multiple ultrastructural differences between filopodia and microspikes are immediately identifiable. Several of these parameters differ from the parameters shown for filament networks, such as lamellipodia, accounting for the bundle architecture. Also, in this case, individual parameters of interest can be plotted with other graph types in order to derive more information. For instance, visualizing the cross-sectional circularity parameter shows a clear reduction in back regions of microspikes, as opposed to their tip regions or to filopodia, likely indicative of less tightly bundled and irregular filament arrangement towards the back of the microspikes (Fig. 4B). This corresponds to reduced values for the base/tip ratio of filament crosssectional density and filament numbers in microspikes, as compared to filopodia (Fig. 4A). Differences in filament spatial arrangement and architecture between filopodia and microspikes are also evident when comparing their angles relative to the axis (Fig. 4C), as well as filament bendiness (Fig. 4D). The extracted quantifications clearly show that filaments in microspikes, as opposed to those in filopodia, are running at higher angles relative to the axis and are more bent, especially in the base of the structure. We implemented an alternative approach for spatial comparison of filament regularity in bundled structures by plotting angles and interfilament distances between filament pairs in cross-sections, based on work by Jasnin et al. (Jasnin et al., 2013) (Fig. 4E and G). This allows identifying the abundance of parallel, regularly arranged filaments within the whole filament population. The presence of such filaments is characteristic of tightly bundled protruding filopodia, where an accumulation of parallel filaments separated by $\sim 10 \mathrm{~nm}$ of interfilament distance is clearly evident (Fig. 4F, marked with white oval shape). Such arrangements are less abundant in the microspikes of our dataset (Fig. 4H). Similarly to the analysis of branched networks, all parameters shown in the linear comparison plot in Fig. 4A can be further analyzed using a large number of plots or using the visualization module of our toolbox to display cross-sections of structures at user-defined positions or characteristics (Suppl. Fig. 3A).

\subsection{Analysis of filaments derived from intact cells}

We so far demonstrated the features of the toolbox with data acquired from cells whose membrane was extracted with a detergent/ surfactant (Triton X-100), a method previously used to increase SNR levels and improve the contrast of filaments (Fäßler et al., 2020a; Vinzenz et al., 2012). We further aimed at applying the toolbox to data derived from non-extracted cells containing also other filaments beyond actin. We first analyzed a publicly available tomogram containing sarcomeres within a native neonatal rat cardiomyocyte (EMD-12572; (Burbaum et al., 2021)). We employed Amira to individually track actincontaining thin filaments and myosin II-based thick filaments and used the visualization module of our toolbox to identify potential differences in their arrangement (Fig. 5A and B). Confirming previous observations (Burbaum et al., 2021; Månsson et al., 2018; Wang et al., 2021), the toolbox was able to identify the regular pattern of $\sim 42-45 \mathrm{~nm}$ interfilament cross-sectional distance between thick filaments, as opposed to the more random distribution between thin filaments (Fig. 5C).

In order to further validate our workflow, we performed cryo-ET on non-extracted B16-F1 cells, and again used Amira for obtaining filament coordinate files (see methods section). We then applied the visualization module of our toolbox to confirm that the toolbox successfully processes data containing both branched filaments of lamellipodial networks and bundled filament arrays of microspikes in cells with preserved plasma membrane (Fig. 5D), where SNR values are lower than in those of cells with no plasma membrane present (Fig. 2). In order to detect quantitatively potential issues arising from decreased SNR values in nonextracted cells, we compared a data set of microspikes in cells with intact plasma membrane to the microspikes data set of membraneextracted cells shown in Fig. 4. Our data indicates no visible differences in the angle, length, bendiness or density of filaments between extracted and non-extracted cells (Fig. 5E). We, however, also want to mention that the quality of filament tracking in non-extracted cells can be variable, something that was more prominently noticeable for lamellipodial filaments, where actin filament tracking in Amira was more robust in detergent extracted tomograms. This again emphasizes the above-stated point that data acquisition settings and further image processing steps need to be fine-tuned for individual specimens and sample conditions.

Overall, we demonstrate that by being downstream in the workflow of the filament segmentation and tracking steps, our computational toolbox remains blind to the sample preparation method used, the composition of filaments or the approach employed to derive coordinates input data.

\subsection{Effects of filament angular distribution with respect to Z-axis on ultrastructural parameters}

Some of the ultrastructural parameters included in the toolbox are dependent on the angular distribution of filaments to the Z-axis. For instance, parameters, such as cross-sectional density or volume fraction, are calculated based on the average number of filaments intersecting a number of cross-sections along the XY-axis of the investigated structure. As shown in Suppl. Fig. 7(A-C), based on the angular distribution of filaments in $\mathrm{Z}$, distinct structures comprised of an identical total number of filaments might display very different quantitative values of crosssectional parameters, such as density, due to the fact that filaments running under acute angles do not simultaneously intersect all crosssections along the length of the structure. It is thus worth noting that depending on the nature of the structure and the organization of filaments within it, interpretation of results should best be performed upon considering multiple ultrastructural parameters and the links between them. One of the strengths of the toolbox is that it allows to quickly derive and display multiple ultrastructural parameters and compare them in order to acquire a clearer idea of how certain features are changed between experimental groups and on the extent of parameter interdependence. Depending on the spatial distribution of filaments and the experimental goal, the user can define a selection of parameters that best characterize the analyzed structure.

\section{Conclusion}

Here we introduce a MATLAB-based computational toolbox, which facilitates the processing and analysis of filament-rich ultrastructural data extracted from cryo-electron tomograms. As a proof-of-principle, we have analyzed a relatively small sample size and compared parameters and experimental samples with obvious differences in filaments distribution. Within this manuscript we did not intend to reveal new biological insights into the actin network architecture, but rather showcase the functionalities of the introduced toolbox. We expect that with increased throughput in data acquisition (Bouvette et al., 2021; Chreifi et al., 2019; Eisenstein et al., 2019), large datasets for a variety of samples can be acquired in a short time, further highlighting the importance to develop ease-of-use tools allowing efficient analysis of the wealth of biological data contained within cellular cryo-electron tomograms. In combination with improved data quality by optimized data acquisition, using for example dual-axis tomography, phase plates, detectors with improved DQE and other means we believe that the real power of the toolbox comes with the time-efficient analysis of large datasets and will allow the detection of subtle ultrastructural differences 
A
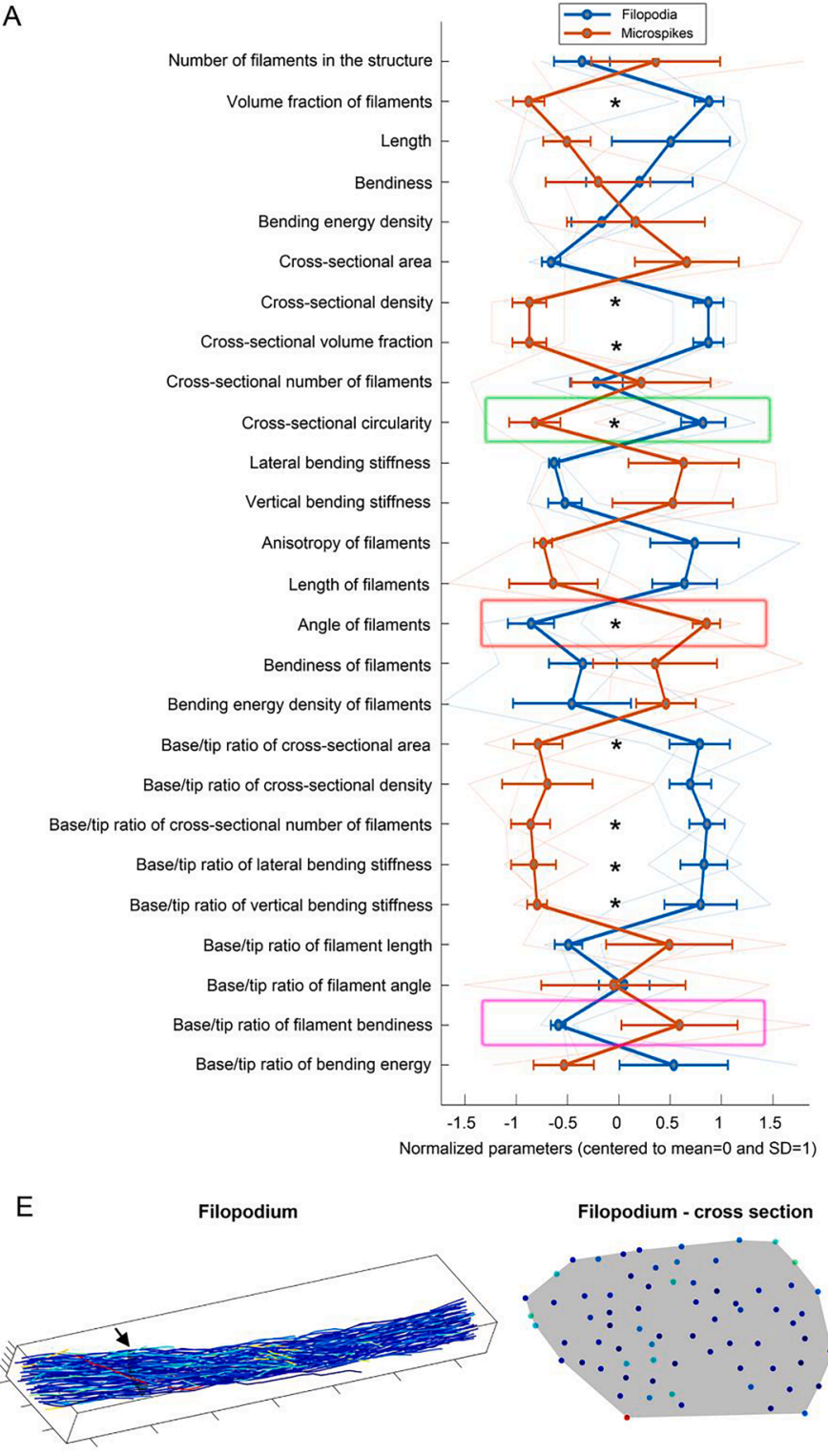

G

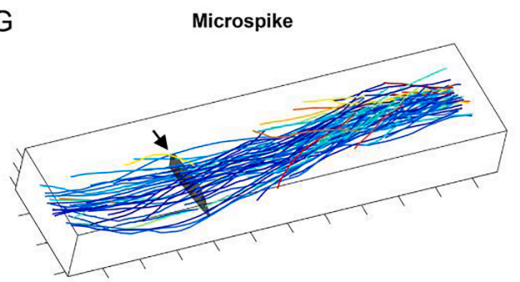

B

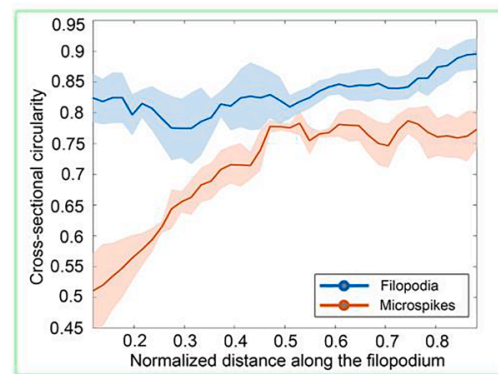

C

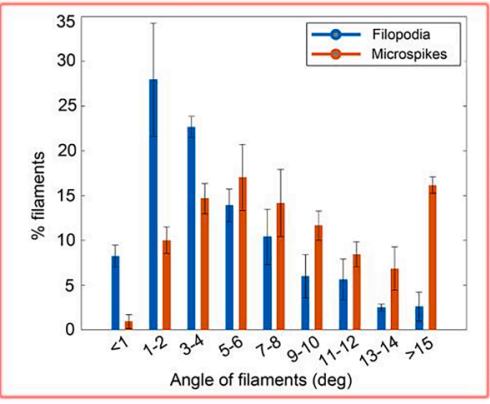

D

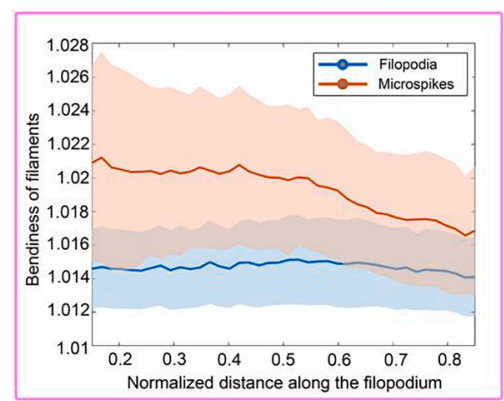

$\mathrm{F}$
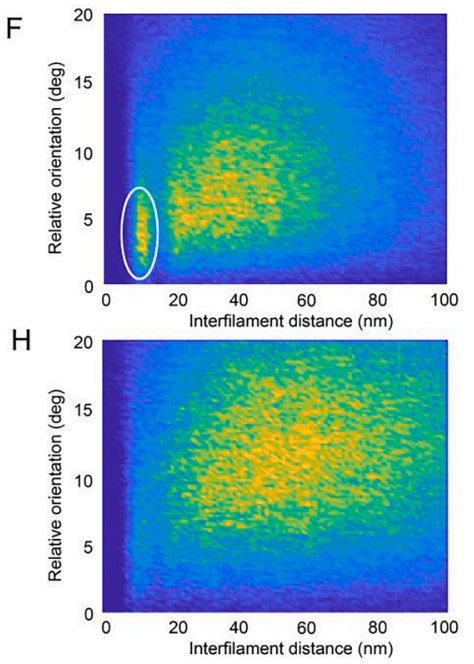

Fig. 4. Analysis of bundled filament arrays. (A) Plotting normalized values of multiple parameters in one graph allows to reveal differences in ultrastructural characteristics of distinct structures. We compared a list of ultrastructural parameters between protruding filopodia and posterior microspikes. Individual parameters have been selected for more detailed comparison. These include: (B) average cross-sectional circularity of filopodia/microspikes along the axis (the transparent outlines indicate standard deviation), (C) angular orientation of filaments in each structure, displayed with a histogram plot of customizable bin numbers and step sizes and (D) local bendiness of filaments along the axis of the structure. The computational toolbox also allows the visualization and extraction of quantitative information on the spatial organization of filaments relative to their neighbors. The integrated visual module was used to first display an example of a (E) protruding filopodium and (G) posterior microspike, where left panels display the analyzed structure with black arrows indicating the location of cross-sectional segments along the axis, and right panels show the cross-sectional distribution of individual filaments color-coded by their local angular orientation to the axis. (F, H) Relating distances between filament pairs (in $\mathrm{nm}$ ) to their relative local orientations (in degrees) demonstrates the presence of a higher number of tightly bundled and parallel oriented filaments within filopodia (indicated with a white oval in F) compared to posterior microspikes (H). All plot options are easily accessible via the GUI-based module. Statistical significance (paired $t$-test, $\mathrm{p} \leq 0.05$ ) between experimental groups is marked with *. The number of tomograms is 3 for both filopodia and microspike groups. 

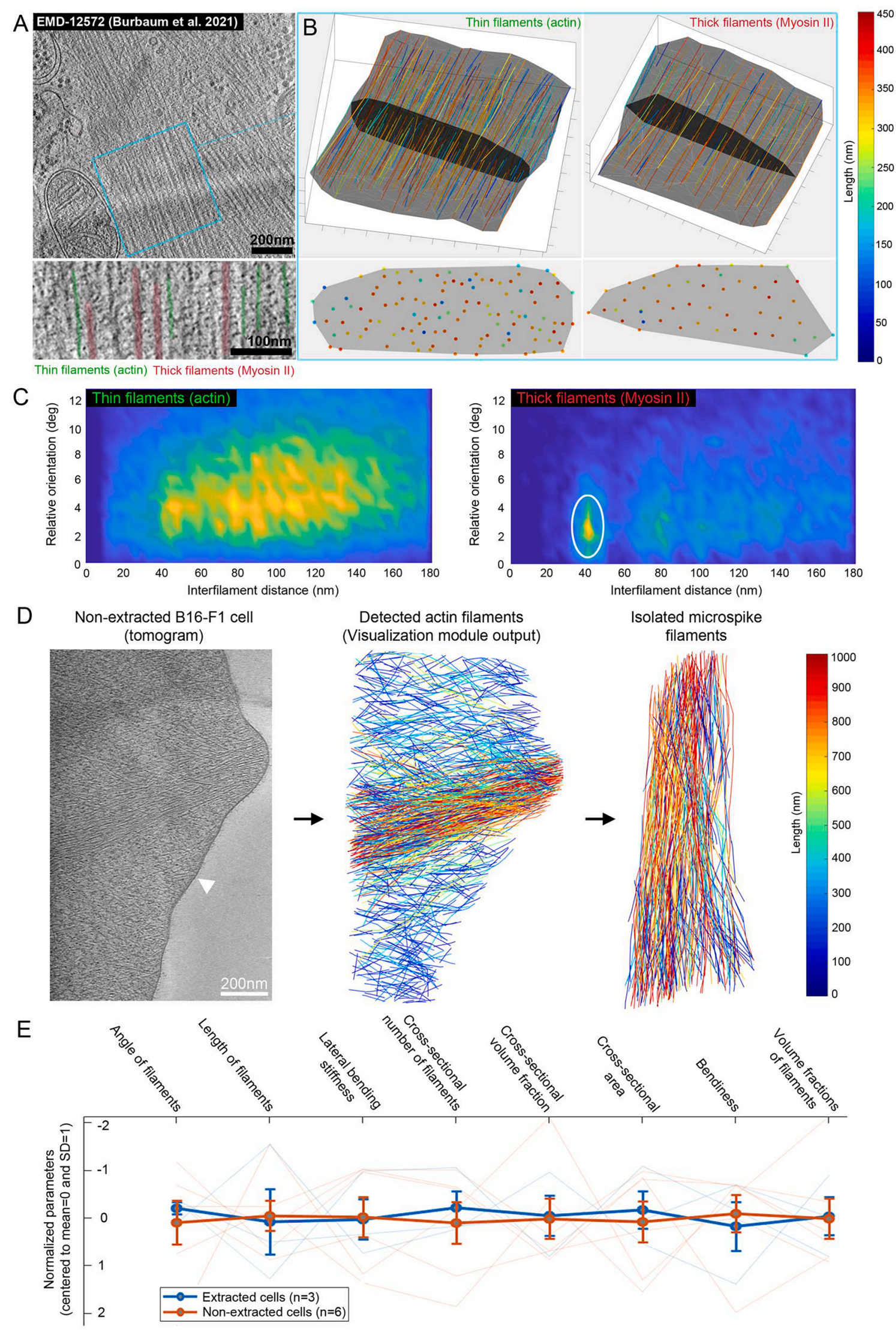

(caption on next page) 
Fig. 5. Analysis of filaments in non-extracted cells. (A) (Left panels): A tomogram slice of a previously published neonatal rat cardiomyocyte lamella (publicly available under EMDB accession code EMD-12572; (Burbaum et al., 2021)), containing thin (actin) and thick (myosin II) filaments. The small bottom panel indicates colorized thin and thick filaments. Amira was used to track thin or thick filaments separately within a specified region (indicated with a cyan rectangle in panel A). (B) The filament tracking results after analysis by the ultrastructural toolbox and displayed with the visualization module. The top panels show the ultrastructural organization of thin filaments and thick filaments. The bottom panels show the cross-section for each filament population, at the indicated position in the top panel. (C) Plotting the distances (in $\mathrm{nm}$ ) between filament pairs to their relative orientations (in degrees) demonstrates distinct cross-sectional arrangements of thin (left panel) and thick (right panel) filaments. Note the density marked with a white oval on the right panel, indicating a pattern of thick filaments being situated regularly at a distance of $\sim 42-45 \mathrm{~nm}$ relative to their neighbors. (D) Left: A slice of a representative tomogram acquired from a vitrified non-extracted cell, with the cell membrane still visibly intact (indicated with a white arrowhead). Filament coordinates were extracted with Amira and subsequently analyzed and plotted with the ultrastructural toolbox. The visualization module of the latter was used to display filaments in the tomogram, colored by length (center panel), from which filaments belonging to the lamellipodial branched network were manually removed with IMOD to only display those belonging to a microspike (right panel). (E) A linear plot comparing ultrastructural parameter values for experimental groups containing microspikes from extracted or non-extracted cells. No differences are detected between the two groups for any of the parameters shown. Data of extracted cells corresponds to microspikes data in Fig. 4. (For interpretation of the references to color in this figure legend, the reader is referred to the web version of this article.)

between experimental conditions, e.g., when comparing multiple genetic knockout clones with mild phenotypes. In such a case, purely visual comparisons, or even smaller scale analysis, might prove to be inconclusive. The wealth of data can be useful for a better understanding of the role of proteins contributing to a given network, or can supplement or enable mathematical modeling approaches of network initiation and maintenance (Falcke, 2016; Zimmermann et al., 2014).

While we developed the toolbox with an emphasis on cellular actin networks and actin-rich cell peripheral structures, it can in principle be used for the analysis of virtually any filamentous network in ET data (or other imaging data) belonging to two different types of ultrastructural assemblies: filament networks (such as branched networks within lamellipodia); and filament architectures, which are aligned in a quasiparallel fashion (such as filopodia or microspikes). There are numerous examples for biological filamentous assemblies for which this analysis is expected to be applicable, e.g., other cytoskeletal elements, such as microtubules or intermediate filaments, and extracellular networks composed of fibrils, such as collagen or fibronectin. The modality of the toolbox allows the implementation of additional features and parameters in the future for investigating other structural configurations or to increase its adaptability for more specialized projects, such as using its results for workflows that aim at determining high-resolution structures of the analysed filaments.

\section{Materials and Methods}

\subsection{Cell culture}

B16-F1 mouse melanoma cells were kindly provided by Klemens Rottner (Technical University Braunschweig, Helmholtz Centre for Infection Research). Cells were grown at $37{ }^{\circ} \mathrm{C}$ and $5 \% \mathrm{CO}_{2}$ and cultured in Dulbecco's modified Eagle's medium (DMEM GlutaMAX, Thermo Fisher Scientific, \#31966047), supplemented with 10\% (v/v) fetal bovine serum (Thermo Fisher Scientific, \#10270106) and 1\% (v/v) penicillin-streptomycin (Thermo Fisher Scientific, \#15070063).

\subsection{Cryo-ET sample preparation and inhibitor treatments}

B16-F1 cells were cultured as described above and seeded onto 200 mesh gold holey carbon grids (R2/2-2C; Quantifoil Micro Tools). Prior to cell seeding, the grids were placed onto a piece of parafilm, glow discharged in an ELMO glow discharge unit (Cordouan Technologies) for $2 \mathrm{~min}$, firmly attached to the bottom of a 6-well flat bottom dish and coated for $1 \mathrm{hr}$ RT with $25 \mu \mathrm{g} / \mathrm{ml}$ laminin (Sigma, L2020) diluted in a buffer containing $150 \mathrm{mM} \mathrm{NaCl}, 50 \mathrm{mM}$ Tris/HCl, $\mathrm{pH}$ 7.5. Grids were gently washed with PBS and cell suspension was pipetted into the well in a slow drop-wise fashion to avoid flipping of the grids.

For CK666 inhibitor treatment, the medium of adherent cells grown overnight onto the EM grids was gently replaced with growth medium supplemented with either $210 \mu$ M CK666 (Sigma Aldrich, \#SML0006) or an equivalent amount of DMSO. An incubation time of $10 \mathrm{~min}$ with the inhibitor was chosen in order to allow CK666 to induce defects in the organization of the actin filaments network in lamellipodia, while not causing the complete retraction of the structure.

Following either overnight growth (for filopodia/microspikes) or overnight growth followed by a 10 min treatment with DMSO/CK666 inhibitor (for lamellipodia), cells were extracted and fixed as previously described (Vinzenz et al., 2012). In brief, grids were incubated for $1 \mathrm{~min}$ at RT in a drop of cytoskeleton buffer (10 mM MES hydrate pH6.2 $(\mathrm{NaOH}), 150 \mathrm{mM} \mathrm{NaCl}, 5 \mathrm{mM}$ EGTA, $5 \mathrm{mM}$ glucose and $5 \mathrm{mM} \mathrm{MgCl2}$ ) supplemented with $0.75 \%$ Triton X-100 (Sigma-Aldrich, \#T8787), $0.25 \%$ glutaraldehyde (Electron Microscopy Services, \#E16220) and $0.1 \mu \mathrm{g} / \mathrm{mL}$ phalloidin (Sigma-Aldrich, \#P2141). Fixation was subsequently performed for 15 minutes at RT by placing the grids in a drop of cytoskeleton buffer containing $2 \%$ glutaraldehyde and $1 \mu \mathrm{g} / \mathrm{mL}$ phalloidin. For deriving a data set of B16-F1 cells with intact (non-extracted) plasma membrane, the same procedure as above was performed, but skipping the extraction step with $0.75 \%$ Triton X-100 and proceeding directly to fixation.

Following extraction and fixation, grids were subjected to back-side blotting (3sec blot time) and vitrification using a Leica GP2 plunger (Leica Microsystems) equipped with a blotting detection sensor and incubation chamber maintaining an environment of $21{ }^{\circ} \mathrm{C}$ and $90 \%$ humidity. Grids were placed into the GP2 incubation chamber and excessive liquid was manually removed with a piece of filter paper by gently touching the side of the grid. Prior to blotting and plunging into liquid ethane $\left(-185^{\circ} \mathrm{C}\right), 3 \mu \mathrm{l}$ of a solution of $10 \mathrm{~nm}$ colloidal gold (AURION Immuno Gold Reagents \& Accessories, Netherlands) coated with BSA in PBS was added onto the grids. Samples were placed in liquid nitrogen storage until imaging.

\subsection{EM Data acquisition}

Tilt-series were either acquired on a Thermo Scientific $300 \mathrm{kV}$ Titan Krios G3i TEM equipped with a BioQuantum post-column energy filter and a K3 camera (Gatan) or on a Thermo Scientific 200 kV Glacios CryoTEM equipped with Falcon 3EC camera. Both microscopes were aligned and operated using the SerialEM package (Mastronarde, 2005).

For data acquired on both microscope systems, the workflow included acquisition of low- and medium-magnification montages for defining regions of interest, followed by high-resolution data acquisition with varying magnification settings and pixel sizes for the different experimental groups (described below).

All filopodia and microspikes data were acquired on a Titan Krios G3i TEM with a total electron dose of $\sim 180 \mathrm{e}^{-} / \AA^{2}$, a tilt range of $-62 /+62$ degrees with 2-degree steps and a defocus of $\sim-3 \mathrm{um}$. Two filopodia and three microspikes were acquired with a pixel size of $2.137 \AA$ (magnification of 42,000x), while one filopodium was acquired with pixel size of $2.676 \AA$ and magnification of $33,000 x$.

Two of the untreated lamellipodia were acquired on Titan Krios Krios G3i TEM with a total electron dose of $\sim 180 \mathrm{e}-/ \AA^{2}$, pixel size $2.137 \AA$ (magnification 42,000x) and defocus of $\sim-4 \mathrm{um}$. 
Two of the untreated lamellipodia and all lamellipodia treated with CK666 were acquired on a Glacios TEM with a total electron dose of $\sim 150 \mathrm{e}^{-} / \mathrm{px}$, tilt series of $-62 /+62$ degrees with 2-degree steps, defocus of $\sim-3 \mathrm{um}$ and pixel size of $3.24 \AA$ (magnification of $45,000 \mathrm{x}$ ).

Data from non-extracted B16-F1 cells was acquired on a Titan Krios with a total dose of $\sim 180 \mathrm{e}-/ \AA^{2}$, a pixel size of $2.676 \AA /$ px and a defocus $\sim-4$ um.

\subsection{EM data processing and extraction of coordinate files}

Pre-processing of acquired tilt series (tilt stack sorting, removal of bad tilts, exposure filtering) was performed with the MATLAB-based Tomoman package (Wan, 2020). Tomogram reconstruction from the filtered tilt series was performed with the IMOD/Etomo software package (version 4.9.12), using weighted back projection.

As illustrated on Suppl. Fig. 1, two different approaches were applied for actin filament vectorization and extraction of filament coordinates from reconstructed tomograms. The first approach involves using tomograms as input for training neuronal networks via the interactive learning and segmentation toolkit Ilastik (Berg et al., 2019) and YAPiC. Actin filaments and background were manually annotated in Ilastik and the Ilastik-derived ilp project files were processed in YAPiC to generate model files, i.e. trained neuronal network instructions for automated segmentation of filaments in a larger dataset. No image processing filters were applied. Separate trainings were performed for lamellipodial networks (with bin8 tomograms) and filopodia/microspikes (with bin4 tomograms). YAPiC-derived model files were used by the same software to generate binary prediction files from reconstructed tomograms, distinguishing between filaments and background.

YAPiC-derived prediction files were processed with MATLAB scripts using the localized radon transform (Winkler et al., 2012) allowing the extraction of files containing XYZ coordinates of points assigned to individual filaments. Cleaning of false-positive filaments was additionally performed via a custom-made Python script eliminating filament pairs within a defined proximity (in pixels) to each other.

Another approach involved processing reconstructed tomograms with the Amira-Avizo software package (version 2020.2), using the "Cylindrical correlation" and "Trace correlation lines" modules. For actin filaments, the following parameter values for the Cylindrical correlation module were set for raw (i.e. header-containing) bin8 tomogram .rec files: Cylinder Length $=500$; Angular Sampling $=5$; Mask Cylinder Radius $=45$; Outer Cylinder Radius $=35$; Inner Cylinder radius $=0$ (all units are in $\AA$ ). The following parameter values were set for the Trace Correlation Lines module: Minimum Seed correlation (tomogram dependent, varying between 80-120); Minimum Continuation Quality $=100$; Direction Coefficient $=0.3$; Minimum Distance $=$ 70; Minimum Length $=350$; Search Cone Length $=500$; Search Cone Angle = 37; Search Cone Minimum Step Size $\%)=10$. For detecting thick myosin-containing filaments from the deposited EMD-12572 tomogram (Fig. 5A and B), the following parameter values for the $\mathrm{Cy}$ lindrical correlation module were set: Cylinder Length $=800$; Angular Sampling = 5; Mask Cylinder Radius = 115; Outer Cylinder Radius = 90; Inner Cylinder radius $=0$ and for Trace Correlation Lines module: Minimum Seed correlation $=80$; Minimum Continuation Quality $=90$; Direction Coefficient $=0.3$; Minimum Distance $=100$; Minimum Length $=800$; Search Cone Length $=800$; Search Cone Angle $=37$; Search Cone Minimum Step Size(\%) $=10$. Segments and point coordinates were extracted as separate excel sheets from Amira-Avizo and reformatted with a custom-made MATLAB-script ("amira_reformat_to_coordinates.m" script provided together with the computational toolbox) in order to obtain a single file per tomogram containing $\mathrm{XYZ}$ coordinates of points assigned to each individual filament.

\subsection{Data pre-analysis cleaning and processing}

Prior to analysis of data files with the computational toolbox, cleaning of unspecific background and false positives was performed. Unspecific background was removed by using the filtering scripts included in the "Supplemental_scripts" folder of the toolbox, excluding all filaments with a length of less than $100 \mathrm{~nm}$ and an angle of less than 75 degrees in $\mathrm{Z}$ axis (as illustrated in Suppl. Fig. 2A). Individual unfiltered filaments, as well as filaments belonging to lamellipodial networks around microspikes, were manually removed with the IMOD software. The "point2model" and "model2point - $c$ " functions were used to reformat respectively .txt coordinate files into IMOD-compatible .mod files or vice versa. For lamellipodia, area of all data files was normalized to $800 \times 800 \mathrm{~nm}$ in $\mathrm{XY}$, by using the cropping script provided in the "Supplemental_scripts" folder of the MATLAB toolbox (see Suppl. Fig. 2B).

\subsection{Software packages used for manuscript assembly and figures preparation}

Coding of the computational toolbox was performed in MATLAB (The MathWorks Inc.). All statistics were performed with the SigmaPlot software (Systat Software Inc.). Figure assembly and preparation was performed with Adobe Photoshop and Adobe Illustrator (Adobe Inc.).

\subsection{Description of ultrastructural parameters}

Since some basic definitions are used repetitively in the description of parameters, we introduce these definitions first.

\section{Reference direction.}

Many of the ultrastructural metrics/parameters described later are dependent on a reference direction. In filamentous networks, such as lamellipodia, it is defined as the direction of a vector pointing towards the leading edge (identical to the axis of lamellipodium or normal direction towards the cell edge). In quasi-parallel filamentous arrays, such as filopodia, it is defined as the direction of a vector starting from the base of the structure to its tip (see axis labeled with " $\mathrm{X}$ " in Suppl. Fig. 8A). Note that this vector is not identical to the axis of the filopodium, which may be a curved line.

\section{Axis and cross sections.}

In the filament networks (e.g. lamellipodia), the axis is a vector pointing towards the leading edge. In the quasi-parallel arrays (e.g. filopodia/microspikes), the axis may also be a curved line from the base to the tip, which follows the curve of the quasi-parallel arrays. In the latter case, the axis is determined using a second order polynomial fitting in the X-Y plane on the data points of all the filaments in the structure. A cross section is defined at a point on the axis, as the plane perpendicular to the direction of the local tangent at that point.

Global and cross-sectional frames of reference.

We define a global frame of reference based on the reference direction. The basis of this frame is defined as follows (see also Suppl. Fig. $8 \mathrm{~A}$ ): $\mathrm{X}$ points towards the reference direction; $\mathrm{Z}$ points in the direction perpendicular to the cell plane (the $X-Y$ plane on which the structure is lying). Note that it is assumed that the filament points in $\mathrm{z}$ indicate a coordinate perpendicular to the cell plane. Finally, $\mathrm{Y}$ is defined as the cross-product of Z and X. Similarly, we define a local cross-sectional frame of reference at every cross section (See Suppl. Fig. $8 \mathrm{~B}$ ). The basis of the local frame of reference are: $\mathrm{x}$ ' is the local tangent vector of the axis; $z$ ' points in the direction perpendicular to the cell plane (similar to Z), and y' is the cross-product of z' and x'. Origin of this local cross-sectional frame of reference is at the center of the mass of the cross section.

Bending energy density (of entire filopodia structures)

Bending energy density for a contour (in this case the axis of the structure) is defined as the normalized sum of the squared local curvature on the contour, i.e.

$$
\frac{1}{L} \int_{0}^{L} \kappa^{2} d l
$$

where $\kappa$ is the local curvature at any point on the contour; $l$ is the 
distance along the contour; $L$ is the contour length.

Volume fraction of filaments.

Ratio of the total volume of all filaments to the total volume of the structure. Each filament is assumed to be a cylinder with the diameter $d$ (default diameter is $7 \mathrm{~nm}$ ). The total volume of filaments is:

$\sum_{i=1}^{N} \pi\left(\frac{d}{2}\right)^{2} l_{i}$

where $N$ is the total number of filaments in the structure and $l_{i}$ is the length of $i$ 'th filament.

Bending energy density of the filaments.

Mean bending energy density of the filaments averaged in the whole actin structure. It is derived similarly to the bending energy density for entire structures parameter, described above. However, as opposed to bending energy of a structure, which is calculated based on the structure axis, bending energy density of the filaments parameter is derived from the contour of individual filaments within the structure.

Anisotropy of filaments.

Mean squared deviation of the angular distribution of the filaments from the uniform angular distribution. $\sum_{\theta=0}^{90}(P(\theta)-1 / 90)^{2}$

where $P(\theta)$ is the probability distribution function of filament angle; $\theta$ is the angle of filaments to the reference direction in X-Y plane.

Cross-sectional volume fraction.

Ratio of the total cross-sectional area of filaments passing a crosssection to the area of the cross-section, averaged for a user-defined number of equidistant cross-sections along the axis. The cross-section of each filament is assumed to be a circle with diameter $d$ (default diameter is $7 \mathrm{~nm}$ ). Thus, the total cross-sectional area of filaments is $n \pi(d / 2)^{2}$, where $n$ is the number of filaments passing the cross section.

Cross-sectional circularity (only for filopodia/microspikes)

Mean cross-sectional circularity of the filopodium averaged across a user-defined number of equidistant cross-sections along the axis. Circularity is defined as the ratio of the cross-sectional area to the area of a circle with the same perimeter.

$\frac{4 \pi . a r e a}{{\text { (perimeter })^{2}}^{2}}$

For a perfect circular cross section circularity equals 1 . It is less than 1 for non circular cross sections.

Lateral bending stiffness (only for filopodia/microspikes)

The moment of inertia of the filaments in a cross section with respect to the z'-axis, averaged across a user-defined number of equidistant cross-sections along the axis. This parameter describes the resistance against lateral bending.

$\sum_{i=1}^{n} y^{\prime}{ }^{2}$

where $\mathrm{n}$ is the number of filaments passing the cross section; $y^{\prime}{ }_{i}$ is distance of the filament $i$ from the z'-axis (Suppl. Fig. 8B).

Vertical bending stiffness (only for filopodia/microspikes)

The moment of inertia of the filaments in a cross section with respect to y-axis, averaged across a user-defined number of equidistant cross sections along the axis. This parameter describes the resistance against vertical bending.

$\sum_{i=1}^{n} z_{i}{ }^{2}$

where $\mathrm{n}$ is the number of filaments passing the cross section; $z_{i}{ }_{i}$ is distance of the filament $i$ from the y'-axis (Suppl. Fig. 8B).

4.8. Parameters describing properties along the axis (Derived from the Plots_Group_Cell_Filopodia/Lamellipodia.m script in the "Properties along axis" menu)

This script illustrates the variation of the properties of the structure along the axis, determined for a user-defined number of equidistant cross-sections along the axis. To calculate the properties that are related to the filaments (length, angle, bendiness, and bending energy density) at a cross-section, we average that property across all the filaments that are passing through the cross-section.

4.9. Parameters describing the spatial arrangement of filaments (Derived from the Plots_Group_Cell_Filopodia/Lamellipodia.m script in the "Configuration of filaments" menu)

We determine the relative distance and orientation of all filament pairs to describe their spatial organization within the structure. To calculate these parameters, a local normal plane at every point along a reference filament is defined (grey rectangle, Suppl. Fig. 8C). This normal plane is perpendicular to the tangent vector of the reference filament (dark blue vector in Suppl. Fig. 8C). The intersections of all other filaments with this plane are then determined. A relative orientation is defined as the angle between the tangent vector of each of these filaments at their intersection with the normal plane (light blue vectors in Suppl. Fig. 8C) and the tangent vector of the reference filament at its intersection point. The interfilament distance is then defined as the distance between the intersection points of each filament and the reference filament with the normal plane. This procedure is repeated for 200 points along each filament and reiterated for every filament in the structure.

\section{Declaration of Competing Interest}

The authors declare that they have no known competing financial interests or personal relationships that could have appeared to influence the work reported in this paper.

\section{Acknowledgements}

This research was supported by the Scientific Service Units (SSUs) of IST Austria through resources provided by Scientific Computing (SciComp), the Life Science Facility (LSF), the BioImaging Facility (BIF), and the Electron Microscopy Facility (EMF). We also thank Victor-Valentin Hodirnau for help with cryo-ET data acquisition. The authors acknowledge support from IST Austria and from the Austrian Science Fund (FWF): M02495 to G.D. and Austrian Science Fund (FWF): P33367 to F.K.M.S.

Code availability

The MATLAB toolbox scripts including a detailed documentation on their usage and example data for processing are available for download via https://schurlab.ist.ac.at/downloads/.

\section{Appendix A. Supplementary data}

Supplementary data to this article can be found online at https://doi. org/10.1016/j.jsb.2021.107808.

\section{References}

Akamatsu, M., Vasan, R., Serwas, D., Ferrin, M., Rangamani, P., Drubin, D.G., 2020. Principles of self-organization and load adaptation by the actin cytoskeleton during clathrin-mediated endocytosis. Elife 9. https://doi.org/10.7554/eLife.49840.

Anderson, K.L., Page, C., Swift, M.F., Suraneni, P., Janssen, M.E.W., Pollard, T.D., Li, R., Volkmann, N., Hanein, D., 2017. Nano-scale actin-network characterization of fibroblast cells lacking functional Arp2/3 complex. J. Struct. Biol. 197 (3), 312-321. https://doi.org/10.1016/j.jsb:2016.12.010.

Berg, S., Kutra, D., Kroeger, T., Straehle, C.N., Kausler, B.X., Haubold, C., Schiegg, M., Ales, J., Beier, T., Rudy, M., Eren, K., Cervantes, J.I., Xu, B., Beuttenmueller, F., Wolny, A., Zhang, C., Koethe, U., Hamprecht, F.A., Kreshuk, A., 2019. ilastik: interactive machine learning for (bio)image analysis. Nat. Methods 16 (12), 1226-1232. https://doi.org/10.1038/s41592-019-0582-9. 
Blanchoin, L., Boujemaa-Paterski, R., Sykes, C., Plastino, J., 2014. Actin Dynamics, Architecture, and Mechanics in Cell Motility. Physiol. Rev. 94 (1), 235-263. https:// doi.org/10.1152/physrev.00018.2013.

Bouvette, J., Liu, H.-F., Du, X., Zhou, Y.e., Sikkema, A.P., da Fonseca Rezende e Mello, J., Klemm, B.P., Huang, R., Schaaper, R.M., Borgnia, M.J., Bartesaghi, A., 2021. Beam image-shift accelerated data acquisition for near-atomic resolution single-particle cryo-electron tomography. Nat. Commun. 12 (1) https://doi.org/10.1038/s41467021-22251-8.

Buckley, G., Gervinskas, G., Taveneau, C., Venugopal, H., Whisstock, J.C., de Marco, A., 2020. Automated cryo-lamella preparation for high-throughput in-situ structural biology. J. Struct. Biol. 210 (2), 107488. https://doi.org/10.1016/j.jsb: 2020.107488.

Burbaum, L., Schneider, J., Scholze, S., Böttcher, R.T., Baumeister, W., Schwille, P., Plitzko, J.M., Jasnin, M., 2021. Molecular-scale visualization of sarcomere contraction within native cardiomyocytes. Nat. Commun. 2021121 12, 1-12. 10.1038/s41467-021-24049-0.

Chen, M., Bell, J.M., Shi, X., Sun, S.Y., Wang, Z., Ludtke, S.J., 2019. A complete data processing workflow for cryo-ET and subtomogram averaging. Nat. Methods 16 (11), 1161-1168. https://doi.org/10.1038/s41592-019-0591-8.

Chreifi, G., Chen, S., Metskas, L.A., Kaplan, M., Jensen, G.J., 2019. Rapid tilt-series acquisition for electron cryotomography. J. Struct. Biol. 205 (2), 163-169. https:// doi.org/10.1016/j.jsb:2018.12.008.

Damiano-Guercio, J., Kurzawa, L., Mueller, J., Dimchev, G., Schaks, M., Nemethova, M. Pokrant, T., Brühmann, S., Linkner, J., Blanchoin, L., Sixt, M., Rottner, K., Faix, J., 2020. Loss of Ena/VASP interferes with lamellipodium architecture, motility and integrin-dependent adhesion. Elife 9. https://doi.org/10.7554/eLife.55351.

Eisenstein, F., Danev, R., Pilhofer, M., 2019. Improved applicability and robustness of fast cryo-electron tomography data acquisition. J. Struct. Biol. 208 (2), 107-114. https://doi.org/10.1016/j.jsb:2019.08.006.

Engel, L., Gaietta, G., Dow, L.P., Swif, M.F., Pardon, G., Volkmann, N., Weis, W.I., Hanein, D., Pruitt, B.L., 2019. Extracellular matrix micropatterning technology for whole cell cryogenic electron microscopy studies. J. Micromechanics Microengineering 29, 1-12. https://doi.org/10.1088/1361-6439/ab419a.

Falcke, M., 2016. Concentration profiles of actin-binding molecules in lamellipodia. Phys. D Nonlinear Phenom. 318-319, 50-57. https://doi.org/10.1016/j. physd.2015.10.019.

Fäßler, F., Dimchev, G., Hodirnau, V.-V., Wan, W., Schur, F.K.M., 2020a. Cryo-electron tomography structure of Arp2/3 complex in cells reveals new insights into the branch junction. Nat. Commun. 11, 6437. https://doi.org/10.1038/s41467-02020286-x.

Fäßler, F., Dimchev, G., Hodirnau, V., Zens, B., Möhl, C., Bradke, F., Schur., F., 2020b. Cryo-electron Tomography Workflows for Quantitative Analysis of Actin Networks Involved in Cell Migration. Microsc. Microanal. 26 (S2), 2518-2519. https://doi. org/10.1017/S1431927620021881.

Fäßler, F., Zens, B., Hauschild, R., Schur, F.K.M., 2020c. 3D printed cell culture grid holders for improved cellular specimen preparation in cryo-electron microscopy. J. Struct. Biol. 212 (3), 107633. https://doi.org/10.1016/j.jsb:2020.107633.

Henson, J.H., Yeterian, M., Weeks, R.M., Medrano, A.E., Brown, B.L., Geist, H.L., Pais, M. D., Oldenbourg, R., Shuster, C.B., Blanchoin, L., 2015. Arp2/3 complex inhibition radically alters lamellipodial actin architecture, suspended cell shape, and the cell spreading process. Mol. Biol. Cell 26 (5), 887-900. https://doi.org/10.1091/mbc. E14-07-1244.

Hetrick, B., Han, M.S., Helgeson, L.A., Nolen, B.J., 2013. Small molecules CK-666 and CK-869 inhibit actin-related protein $2 / 3$ complex by blocking an activating conformational change. Chem. Biol. 20 (5), 701-712. https://doi.org/10.1016/j. chembiol.2013.03.019.

Jasnin, M., Asano, S., Gouin, E., Hegerl, R., Plitzko, J.M., Villa, E., Cossart, P., Baumeister, W., 2013. Three-dimensional architecture of actin filaments in Listeria monocytogenes comet tails. Proc. Natl. Acad. Sci. 110 (51), 20521-20526.

Jasnin, M., Beck, F., Ecke, M., Fukuda, Y., Martinez-Sanchez, A., Baumeister, W., Gerisch, G., 2019. The Architecture of Traveling Actin Waves Revealed by CryoElectron Tomography. Structure 27 (8), 1211-1223.e5. https://doi.org/10.1016/j. str.2019.05.009.

Jasnin, M., Crevenna, A.H., 2016. Quantitative Analysis of Filament Branch Orientation in Listeria Actin Comet Tails. Biophys. J. 110 (4), 817-826. https://doi.org/ 10.1016/j.bpj.2015.07.053.

Jasnin, M., Ecke, M., Baumeister, W., Gerisch, G., 2016. Actin Organization in Cells Responding to a Perforated Surface, Revealed by Live Imaging and Cryo-Electron Tomography. Structure 24 (7), 1031-1043. https://doi.org/10.1016/j. str.2016.05.004.

Kage, F., Winterhoff, M., Dimchev, V., Mueller, J., Thalheim, T., Freise, A., Brühmann, S., Kollasser, J., Block, J., Dimchev, G., Geyer, M., Schnittler, H.J., Brakebusch, C., Stradal, T.E.B., Carlier, M.F., Sixt, M., Käs, J., Faix, J., Rottner, K., 2017. FMNL formins boost lamellipodial force generation. Nat. Commun. 10.1038/ ncomms14832.
Månsson, A., Ušaj, M., Moretto, L., Rassier, D.E., 2018. Do Actomyosin Single-Molecule Mechanics Data Predict Mechanics of Contracting Muscle? Int. J. Mol. Sci. 2018, Vol. 19, Page 1863 19, 1863. 10.3390/IJMS19071863.

Martins, B., Sorrentino, S., Chung, W.-L., Tatli, M., Medalia, O., Eibauer, M., 2021. Unveiling the polarity of actin filaments by cryo-electron tomography. Structure 29 (5), 488-498.e4. https://doi.org/10.1016/j.str.2020.12.014.

Mastronarde, D.N., 2005. Automated electron microscope tomography using robust prediction of specimen movements. J Struct Biol 152 (1), 36-51. https://doi.org/ 10.1016/j.jsb:2005.07.007.

Mastronarde, D.N., 1997. Dual-axis tomography: an approach with alignment methods that preserve resolution. J Struct Biol 120 (3), 343-352. https://doi.org/10.1006/ jsbi.1997.3919.

Mastronarde, D.N., Held, S.R., 2017. Automated tilt series alignment and tomographic reconstruction in IMOD. J. Struct. Biol. 197 (2), 102-113. https://doi.org/10.1016/ j.jsb:2016.07.011.

Molinie, N., Gautreau, A., 2018. The Arp2/3 Regulatory System and Its Deregulation in Cancer. Physiol. Rev. 98 (1), 215-238. https://doi.org/10.1152/ physrev.00006.2017.

Mueller, J., Szep, G., Nemethova, M., de Vries, I., Lieber, A.D., Winkler, C., Kruse, K., Small, J.V., Schmeiser, C., Keren, K., Hauschild, R., Sixt, M., 2017. Load Adaptation of Lamellipodial Actin Networks. Cell 171 (1), 188-200.e16. https://doi.org/ 10.1016/j.cell.2017.07.051.

Narita, A., Mueller, J., Urban, E., Vinzenz, M., Small, J.V., Maéda, Y., 2012. Direct determination of actin polarity in the cell. J. Mol. Biol. 419 (5), 359-368. https:// doi.org/10.1016/j.jmb.2012.03.015.

Rigort, A., Günther, D., Hegerl, R., Baum, D., Weber, B., Prohaska, S., Medalia, O., Baumeister, W., Hege, H.-C., 2012. Automated segmentation of electron tomograms for a quantitative description of actin filament networks. J. Struct. Biol. 177 (1), 135-144. https://doi.org/10.1016/j.jsb:2011.08.012.

Schorb, M., Haberbosch, I., Hagen, W.J.H., Schwab, Y., Mastronarde, D.N., 2019. Software tools for automated transmission electron microscopy. Nat. Methods 16 (6), 471-477. https://doi.org/10.1038/s41592-019-0396-9.

Schur, F.K., 2019. Toward high-resolution in situ structural biology with cryo-electron tomography and subtomogram averaging. Curr. Opin. Struct. Biol. 58, 1-9. https:// doi.org/10.1016/j.sbi.2019.03.018.

Svitkina, T.M., Bulanova, E.A., Chaga, O.Y., Vignjevic, D.M., Kojima, S. ichiro, Vasiliev, J.M., Borisy, G.G., 2003. Mechanism of filopodia initiation by reorganization of a dendritic network. J. Cell Biol. 10.1083/jcb.200210174.

Toro-nahuelpan, M., Zagoriy, I., Senger, F., Blanchoin, L., Mahamid, J., 2019. Tailoring cryo-electron microscopy grids by photo-micropatterning for in-cell structural studies 1-16.

Urban, E., Jacob, S., Nemethova, M., Resch, G.P., Small, J.V., 2010. Electron tomography reveals unbranched networks of actin filaments in lamellipodia. Nat. Cell Biol. 12 (5), 429-435. https://doi.org/10.1038/ncb2044.

Vinzenz, M., Nemethova, M., Schur, F., Mueller, J., Narita, A., Urban, E., Winkler, C., Schmeiser, C., Koestler, S.A., Rottner, K., Resch, G.P., Maeda, Y., Small, J.V., 2012. Actin branching in the initiation and maintenance of lamellipodia. J. Cell Sci. https://doi.org/10.1242/jcs.107623.

Volkmann, N., 2004. An approach to automated particle picking from electron micrographs based on reduced representation templates. J. Struct. Biol. 145 (1-2), 152-156. https://doi.org/10.1016/j.jsb:2003.11.026.

Wan, W., 2020. williamnwan/TOMOMAN: TOMOMAN 08042020. 10.5281/ ZENODO.4110737.

Wang, Z., Grange, M., Wagner, T., Kho, A.L., Gautel, M., Raunser, S., 2021. The molecular basis for sarcomere organization in vertebrate skeletal muscle. Cell 184 (8), 2135-2150.e13. https://doi.org/10.1016/j.cell.2021.02.047.

Weber, M., Wojtynek, M., Medalia, O., 2019. Cellular and Structural Studies of Eukaryotic Cells by Cryo-Electron Tomography. Cells 8 (1), 57. https://doi.org/ 10.3390/cells8010057.

Winkler, C., Vinzenz, M., Small, J.V., Schmeiser, C., 2012. Actin filament tracking in electron tomograms of negatively stained lamellipodia using the localized radon transform. J Struct Biol 178 (1), 19-28. https://doi.org/10.1016/j.jsb:2012.02.011.

Winter, S.L., Chlanda, P., 2021. Dual-axis Volta phase plate cryo-electron tomography of Ebola virus-like particles reveals actin-VP40 interactions. J. Struct. Biol. 213 (2), 107742. https://doi.org/10.1016/j.jsb:2021.107742.

Xu, X.-P., Page, C., Volkmann, N., 2015. Efficient Extraction of Macromolecular Complexes from Electron Tomograms Based on Reduced Representation Templates BT - Computer Analysis of Images and Patterns, in: Azzopardi, G., Petkov, N. (Eds.), . Springer International Publishing, Cham, pp. 423-431.

Zachs, T., Schertel, A., Medeiros, J., Weiss, G.L., Hugener, J., Matos, J., Pilhofer, M., 2020. Fully automated, sequential focused ion beam milling for cryo-electron tomography. Elife 9. https://doi.org/10.7554/eLife.52286.

Zhang, P., 2019. Advances in cryo-electron tomography and subtomogram averaging and classification. Curr. Opin. Struct. Biol. 58, 249-258. https://doi.org/10.1016/j. sbi.2019.05.021.

Zimmermann, J., Falcke, M., Aspenstrom, P., 2014. Formation of transient lamellipodia. PLoS ONE 9 (2), e87638. https://doi.org/10.1371/journal.pone.0087638. 